\title{
A context-driven geospatial web service approach towards the design of situational awareness maps
}

\author{
Sin-Yi Ho ${ }^{a}$, Jung-Hong Hong ${ }^{b}, *$ \\ ${ }^{a}$ Undergraduate student, Department of Geomatics, National Cheng Kung University, Taiwan, f64041208@ gmail.com \\ ${ }^{b}$ Department of Geomatics, National Cheng Kung University, Taiwan, junghong@ mail.ncku.edu.tw \\ * Corresponding author
}

\begin{abstract}
The recent development of internet-based map services has dramatically change how geospatial information is presented to users, novice or professional, for improving the quality and convenience of daily lives. Especially with the innovated internet and computer graphic technology, current map services are designed with comprehensive cartographic considerations and can be simultaneously accessed by multiple clients, with which users can metaphorically establish their understanding towards the phenomena in reality, regardless of where they are. Despite location-based services become increasingly popular, the serviced contents are nevertheless often "static" and the illustration seldom or even fail to adapt to the needs of presenting continuously or periodically changing situations in the real world. From the perspective of intuitive map reading, we argue it is necessary to include the needs of "situational awareness" into the design of map services, so as to improve the human computer interactions in the clients' application environments. A context-driven approach for the design and mashup of map services are proposed. We first identify three types of map services, namely, reference map services, application-oriented map services and atomic map services. The context of each map services are analysed based on the considerations of theme, time, weather and transportation and described by a set of pre-defined "keywords." These standardized keywords enable application developers to define rules to select map services with correct contexts and mashup them to meet particular situation needs, e.g., a rainy day map or an emergency medical map services. As the selected map services are already defined with context considerations, their mashup results would be automatically adjusted according to the situations acquired from the clients. By enriching the situational context and enhancing the transparent access of individual map services, a flexible and powerful bridging relationship can be built between the application developers and domain service providers. The proposed approach is hence not only an innovated thinking for designing map services, but should also be seen as a crucial strategy for facilitating interoperable application environments for the ecosystem of the National Spatial Data Infrastructure.
\end{abstract}

Keywords: situational-awareness, map service, context-driven, geospatial web service

\section{Introduction}

The development of GIS platforms has evolved from desktop in the earlier years to Web Geographic Information Systems (WebGIS) now. Especially in recent years, after the rapid growth of Internet services and mobile device technology, the advantage of almost all users have one mobile device makes everyone enjoy the convenience of various GIS-based applications. This further triggers the vigorous growth of the electronic government and geographic information industries. To attract clients to use their applications, service providers nowadays must spend tremendous efforts to not only provide customized and quality services, but also continuously come out new ideas to improve their services. Since GIS-based applications often require a large amount of information on different themes, take the best advantages of the data from other providers has become an important and necessary task. This is especially true for the map services, as application developers may have little or even none knowledge about the geographic data owned by other providers, so they are most likely preferred to access data that is already presented by maps. Among all the map services, the most widely mentioned service is the basemap service, which typically includes the cartographic illustration of a wide range of topographic phenomena on the surface of the earth. The publication of basemap services enables users to instantly access ready-to-use maps about their neighborhood regardless of where they are. With a global or national basemap service, application developers can further take advantages of this locationbased nature by overlaying thematic map services to develop customized applications (Walawender and Davis, 2010). An effective collaborated application framework of map services, which gather map services from all the participating organizations in an organized fashion, can bring extremely positive benefits to the continuous growth of the GIS market. From the government perspective, this is also an essential work for promoting the sharing mechanism of the national spatial data infrastructure (NSDI).

In this collaborated framework, the success of developed applications is largely dependent on if developers can take 
good use of the map services provided by other professional agencies. How the accessed map service is designed and used are, however, totally controlled by the providers, meaning decisions made by the providers may restrict the subsequent use of the accessed map services for further value-added applications. For example, clients have no way to change the map symbols presented by the imagery map tile services. Hence the cartographic decisions made by the service provides will restrict the possible extended use on the clients' side. Many providers simply choose to publish data in its original state and leave the interpretation and processing of the data to the application developers and even general public. The lack of understanding about the acquired data becomes huge obstacles for advanced developments and increase the risks of wrong use. While encouraging more organizations to share their resources, it is indeed necessary to ask service providers to examine how their map services are designed and provided.

First appeared in 2004, the concept of "mashup" is defined as the process of mixing different types of software and different sources data to form a new effect (Michael Batty et al, 2010). In 2013, Buqing Cao et al. defined that mashups, which are Web applications created by combining two or more services, are becoming a hot research topic (Buqing Cao et al., 2013). In the field of geographic information system, "map mashup" is a web page or application that uses and combines data, presentation or functionality from two or more sources to create new services from Wikipedia. The mashup architecture integrates the data and information from various different sources such as open APIs, web services, etc. and presents it as a new application (Harish Chandra Karnatak, 2012). Successful mashup can generate service contents with the effect of synergy, meaning aggregated or integrated results that cannot be provided by a single service. Therefore, the more variety of services available, the more possibility to design mashup services to address different application needs. As available services may be from different sources, the mashup of services requires to consider how to conquer the heterogeneity and interoperability issues. The series of standards from OGC (Open Geospatial Consortium) has facilitated an open sharing environment based on geospatial web services and remove the barriers of data exchange. Although the number of map services following OGC standards has been steadily increasing in recent years, we must recognize that each map service standard has its own characteristics and limitations (OGC, 2005). While determining how to distribute domain data via open web services, it should be the responsibility for the source providers to carefully examine the purposes and possible applications, such that their services can be easily consumed by the developed applications. For example, a restaurant map published by WMS is good for showing where the restaurants are, but it cannot indicate the open hours for individual restaurants. Hence it is good for showing the geographic distribution of restaurants (as most reference map services do), but not appropriate for visually presenting which restaurant is opened now. If the service providers are reminded the information about the open hours of restaurants may be needed, their choices of packaging restaurant data and map service standards may be rather different. The improved design of map services can also bring positive benefits to the mashup map services, as there would be more alternative map services that can be readily accessed to meet particular application needs.

As the phenomena in reality may continuously changes over time, it is clearly advantageous to develop "live" map services, meaning the map services are designed in a way to be automatically aware of the changing situations and capable of adjusting the serviced contents and illustrations to aid clients' decision making. We argue the key challenge is to choose the map service with the correct context. Context is defined as "any information that can be used to characterize the situation of a person, place, or object that is considered relevant to the interaction between a User and application" (Blefari Melazzi et al, 2007; José Rodríguez et al, 2013). From the map service perspective, every map service shall have its own context, e.g., content, theme, visualization, usage, quality and even limitations. The mashup of map services must be based on the correct understanding about the contexts of the selected map services, otherwise the mashup results will not have the correct context. The core of this research includes two major strategies. First of all, every map services must have specific contexts described by standardized vocabularies according to their distinguished situational-awareness characteristics. Secondly, application developers can define rules to flexibly select and dynamically mashup various map services based on the standardized vocabularies. Based on this context-driven approach, the interoperable communications between service providers and application developers can be also improved.

The remaining of this paper is organized as follows. Section 2 introduces the proposed context-driven framework of map services and the situational-aware mashup strategies. The discussion also covers the interactions between different participants in the servicebased ecosystem. Section 3 demonstrates how the three basic types of map services are designed and described. Finally, section 4 concludes our major findings and suggest some future works.

\section{System Architecture}

This section explains how to incorporate the requirements of situational-awareness into the newly introduced application frameworks based on geospatial web services with specific contexts.

\subsection{The ecosystem of Service-based Environment}

In the ecosystem of web service application environment, three types of participants are identified: service providers, application developers and clients. In addition to the specific roles each type of participants plays, the interactions between different types of participants are also important for the success of the framework. 
(1) Service providers

Any government agencies, private sectors, or individual persons can be service providers, as long as they own a dataset and have the capability of publishing the dataset. As service providers have thorough control about how data is designed and distributed, it is their responsibility to formally and unambiguously provide the necessary information about the services to the potential users. Dependent on the purposes and intended application of the services, the design strategies may be rather different. For example, basemap services are mainly used for visual reference purpose, so cartographic design is necessary. On the other hand, phenomena changes with time will additionally demand the map service to continuously change its illustration with time, so WMTS may be not a good choice for publishing such data. From the perspective of NSDI, all the developed services should register to the sharing mechanism.

(2) Application developers

The ultimate goal of the application developers is to design applications that meet clients' customized needs. Despite application developers may have certain types of domain data, but are often required to access existed services registered in the sharing mechanism to reduce spending and improve quality. Mashup operation is widely used to generate interesting outcomes based on the selected services. The key challenge for the application developers is therefore to find, understand, select and mashup the services according to their requirement analysis. This demand is practically impossible if service providers fail to unambiguously explain their services.

(3) Clients

Clients interact with the chosen applications to express their requests and receive responses. Both requests and responses are preferred to be implemented in a smart and friendly interface with minimum human involvement. For example, a beautiful illustration in the map interface that meets the direct visual inspection needs is definitely welcome by most of the clients. (Figure 1)

\subsection{Context-driven services}

For the map services, we further identify three types of services, namely, atomic map services, applicationoriented map services and basemap services. Each type of map services has its preferred application scenarios, as summarized in Table 1:

\begin{tabular}{|c|c|c|}
\hline $\begin{array}{l}\text { Types of } \\
\text { service }\end{array}$ & descriptions & Application scenarios \\
\hline $\begin{array}{l}\text { Atomic data } \\
\text { services }\end{array}$ & $\begin{array}{l}\text { Individual } \\
\text { theme of } \\
\text { dataset. }\end{array}$ & $\begin{array}{ll}\text { - Working as an } \\
\text { independent layer } \\
\text { in applications. } \\
\text { Either presented as } \\
\text { a geo-reference } \\
\text { images or a feature } \\
\text { dataset. }\end{array}$ \\
\hline $\begin{array}{l}\text { Application- } \\
\text { oriented } \\
\text { map } \\
\text { services }\end{array}$ & $\begin{array}{l}\text { Mashup of } \\
\text { various } \\
\text { themes of } \\
\text { map } \\
\text { services for } \\
\text { specific } \\
\text { purpose. }\end{array}$ & $\begin{array}{l}\text { Readily available } \\
\text { for the selected } \\
\text { application } \\
\text { scenario. } \\
\text { Some of them } \\
\text { have cartographic } \\
\text { design for direct } \\
\text { visualization. }\end{array}$ \\
\hline $\begin{array}{l}\text { Basemap } \\
\text { services }\end{array}$ & $\begin{array}{l}\text { General } \\
\text { reference } \\
\text { map } \\
\text { purpose. }\end{array}$ & $\begin{array}{l}\text { Provide } \\
\text { neighborhood } \\
\text { information for the } \\
\text { point of interests. } \\
\text { Cartographic } \\
\text { design for direct } \\
\text { visualization. }\end{array}$ \\
\hline
\end{tabular}

\section{User Requirements}

The system detects the current situation and receive the user's requirements, through the rule to reply the best situation awareness map.

\section{Data Service}

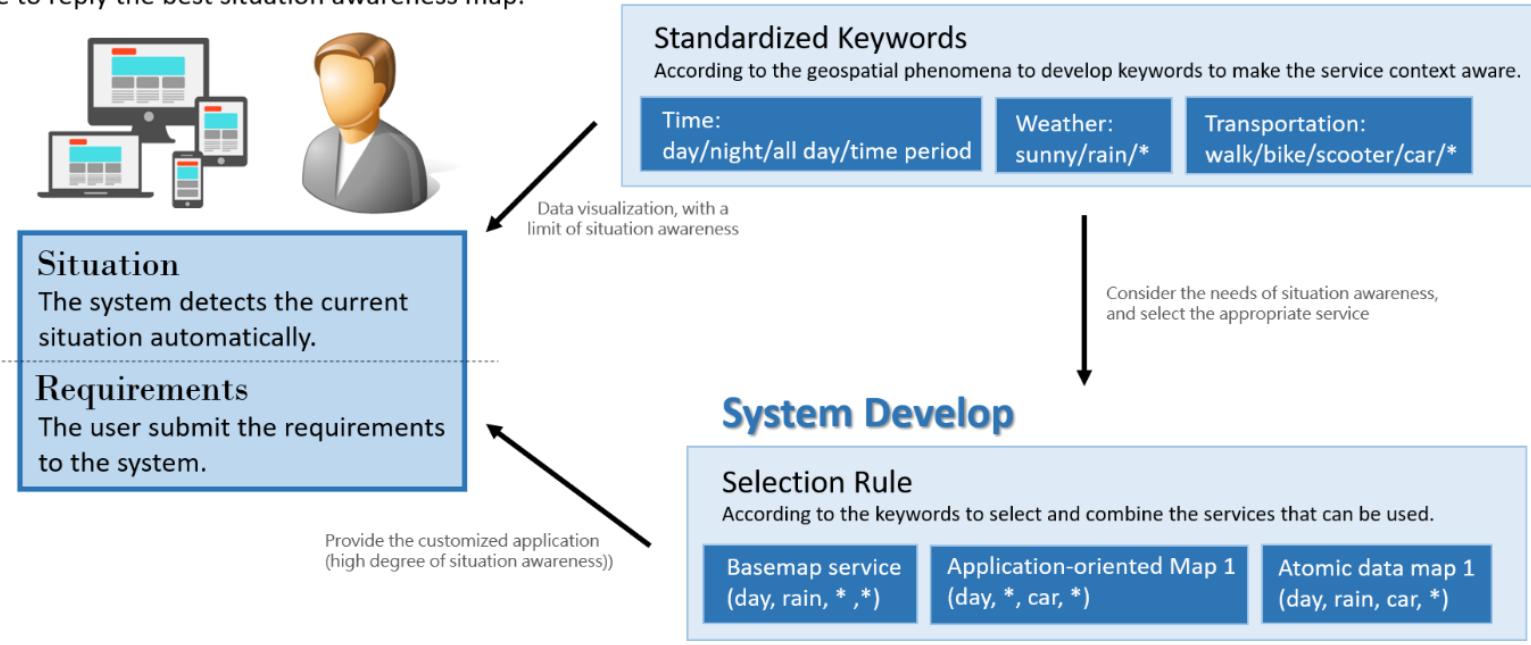

Figure 1. Three types of participants in the service-based ecosystem. 
The atomic map services will serve as the basis for developing application-oriented services or basemap services, as both of them requires multiple themes of data. As mentioned in the section 1, basemap services are widely used in current GIS-based applications and often considered as the core or essential map service for the NSDI.

After choosing the types of map services, service providers have to further consider which OGC standards to follow to publish the services. Table 2 summarizes the major characteristics of the commonly used OGC map service standards. Each standard has its preferred application scenarios as well as the limitations.

\begin{tabular}{|l|l|}
\hline $\begin{array}{l}\text { Service } \\
\text { standard }\end{array}$ & Application \\
\hline WMS & $\begin{array}{l}\text { Response with a geo-referenced images, } \\
\text { mainly used for visualization purpose } \\
\text { (map overlay). } \\
\text { Use SLD to publish multiple styles of map } \\
\text { services. }\end{array}$ \\
\hline WMTS & $\begin{array}{l}\text { Response with a number of map tiles, } \\
\text { mainly used for basemap service. } \\
\text { Imagery map tiles are produced in } \\
\text { advance with specific styles. }\end{array}$ \\
\hline WFS & $\begin{array}{l}\text { Response with feature-based data, mainly } \\
\text { used for further query or analysis purpose. } \\
\text { Further visualization design is necessary. }\end{array}$ \\
\hline Vector tile & $\begin{array}{l}\text { Similar to imagery map tile services, but } \\
\text { with more flexibility of customizing the } \\
\text { visualizations. }\end{array}$ \\
\hline
\end{tabular}

Table 2. Major characteristics of the OGC standards.

Every map service shall have its own distinguished context and this information must be made available to all the potential users. To aid the understanding of available services, we propose to use standardized "keywords" to describe the context of services. Three types of keywords are proposed:
(1) Theme: Theme keywords are used to describe the theme of the data the map service publishes. It can be keywords specifically used to describe a particular type of phenomena (e.g., river), or to describe the aggregation or mashup of multiple themes of phenomena (e.g., emergency medical map or topographic map). As every application shall have its own requirements of data, theme keywords often serve as the first criteria for choosing map services.

(2) Situation: Situation keywords are used to explain the situations the services can be used. This is especially useful for developing services whose contents whose content must be adjusted under different circumstances. For example, we may publish two services respectively related to the phenomena in the daytime and night, and use different situation keywords to explain their differences. This enables clients to visually inspect two styles of maps at different time. The more situations considered, the more situation keywords a service may have and the better customization can be reached.

(3) Visualization: Visualization keywords are mainly used to indicate if the application developers are allowed to modify the visualization of the accessed map services. This is usually dependent on the chosen map service standard. For example, the georeferenced images from the service of WMTS (imagery map tiles) and WMS are often only used for direct visualization, while the visualization of feature-based data from the services of WFS can be further arranged or modified.

The context of individual map service is therefore described by a number of keywords. To be further linked to the selection of map service, the set of vocabularies for each type of keywords must be standardized (refer to the discussion in section 2.3). As the context determine how a map service is designed, we further propose an analysis

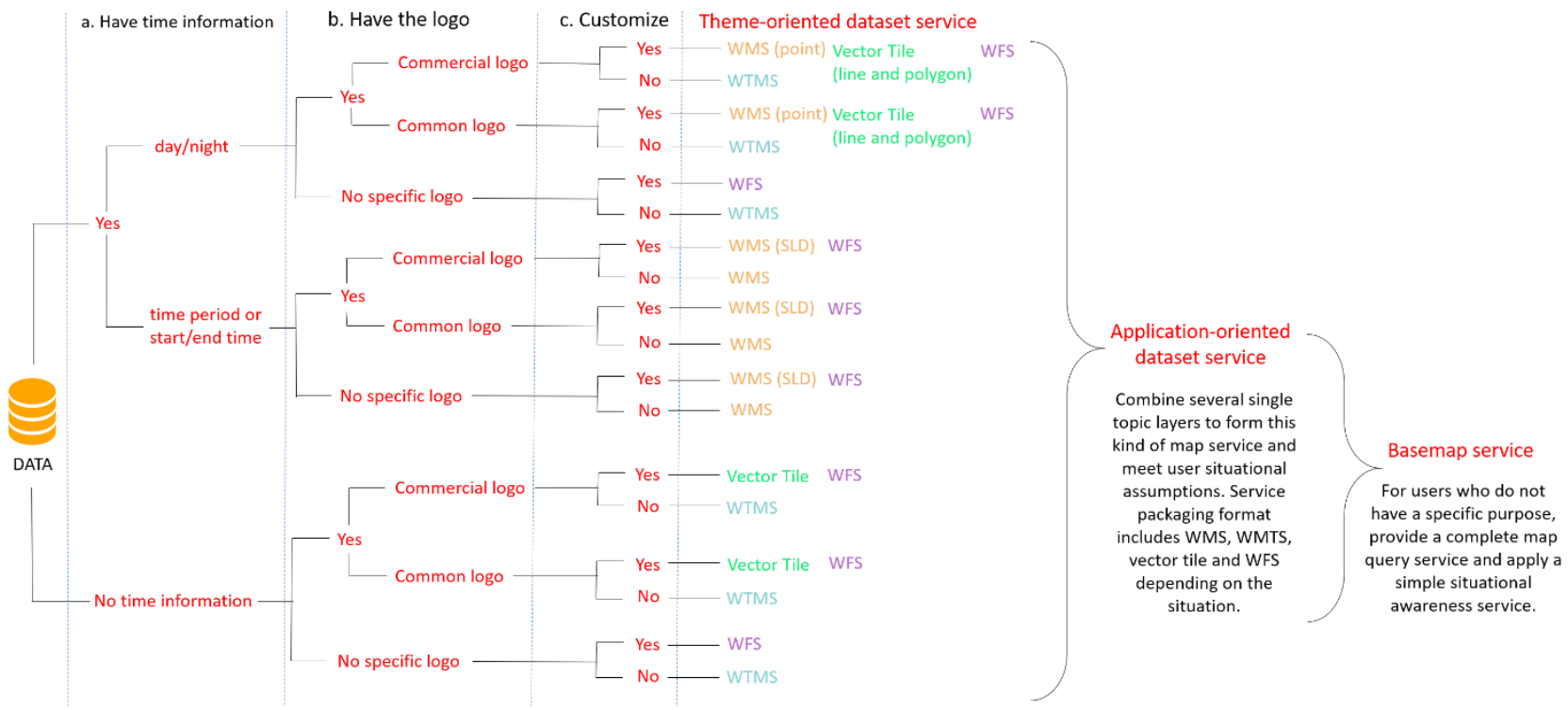

Figure 2. Analysis procedures for designing context-driven services. 
procedure for designing map services based on the considerations of situations and visualization (Figure 2). The proposed procedure starts with the analysis of the temporal issue of the presented phenomena. Service providers are required to determine if the serviced data and its visualization required to change with time. The second factor considered is if the presented phenomena has logo that are widely recognized by the clients. For map services with cartographic design, it is preferred to directly publish data with map symbols based on the logo clients are already familiar with. For example, the convenience store chain can publish the location of its branch store via WMS by using its logo as map symbols and reduce the extra cartographic design works for the application developers. As the use of WMS may restrict the possibility of changing the presentation styles, the service providers may alternatively consider to publish feature-based data via WFS as well. Dependent on the outcomes of the analysis, different map service standards are proposed. It is possible for an individual theme of data to be published by a number of atomic services with different contexts after analysis.

\subsection{Situation keywords}

As the major goal of this research is about the "situational awareness" of the applications, situation keywords play a dominant for selecting services. Three types of situation keywords are discussed in this research: time, weather and transportation. Each type of situation keywords requires to select a set of vocabularies that are used to describe the contexts of the map services:

(1) Time: Ideally speaking, all map services should provide temporal information about when the services can be used. The proposed keywords include day, night, all day and time period (with start and end time). The meaning of these four keywords are exclusive with each other. For example, the keyword of "day" implies the described phenomena is only available in the daytime, while the keyword of "all day" implies the described phenomena is always available. Since these time keywords are used to describe when the presented phenomena is valid, application developers can directly select the "right" map services according to the time of service. As time goes by, the developed application may need to "switch" to another map service to ensure the context can meet the changing situations.

(2) Weather: The weather condition is another situation that may impact the content of the service. Different from the time keywords, weather keywords are used to indicate the map services can be used, but not limited to, the described situations. Two keywords are discussed in this research: sunny and rainy. For the case of rainy days, any map services that can provide useful reference information can be described by the keyword of "rainy." For example, nearby stores selling umbrella (can be stores from different atomic services) or shelters of buildings from raining are both qualified map services.

(3) Transportation: The serviced content is also dependent on the types of transportation chosen. The keywords include car, scooter, bicycle, walk. When considering moving from one place to another, the path for cars and bicycles may be rather different. Without context information about the transportation, the decisions may contain unexpected risk, for example, using bicycle road network data for planning the routes for cars.

The above three types of keywords help application developers to understand and choose the map services with the "right" context for the intended applications. The types of situations can be further expanded to enrich the context of the available map services in the future.

\subsection{Rules}

With the context described by the standardized keywords, application developers can directly select map services with the right contexts for their applications. They can even develop new services with the mashup of multiple map services. Rules are defined on the basis of the themes of datasets:

data themes for the application(context)

$$
\begin{aligned}
& =\text { theme } 1 \text { (context) }+ \text { theme } 2 \text { (context) } \\
& + \text { theme } 3 \text { (context) }+\cdots+\text { theme } n(\text { context })
\end{aligned}
$$

In addition to the consideration about the theme keywords, the context of developed application and the contexts of all the selected services must be also compatible. For example, to mashup two atomic services respectively referred to day and night will not yield meaningful results. It is also possible to design application-oriented map services by defining rules to select multiple atomic services. The context of the new services must be based on the contexts of the selected services. Using the rainy map service in day as an example, the map services of convenience store, household life store and entertainment are all regarded as places that can provide related help. Only map services whose context is about "day" or "all day" are selected.

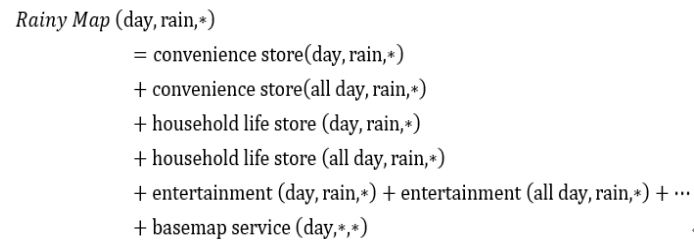

\subsection{Client side}

To make the situation-awareness mechanism works, the applications must continuously acquire information from the clients to update the current situations. Some of the information may be directly acquired from clients' hardware devices (e.g. locations, time), while some other types of information may require clients to submit to the applications (e.g., transportation, weather), so that applications can provide customized services accordingly. Although it is not necessary for all clients to be familiar with the keywords that describe the context, keywords with semantic consideration are certainly beneficial for 
better human computer interactions. In addition to the use of keywords, further processing the received data on the applications is sometimes necessary. For example, the format of the time information acquired from the clients is recorded as date-time, and is later transformed to be referred to the time keywords according to the rules the application developers specify (e.g., day and night).

\section{Results and Analysis}

This section uses real examples to demonstrate how to develop situation-aware map services. Every service has its context following the procedure discussed in section 2.2 and 2.3. The contexts of atomic map services can serve as the basis for determining the contexts of the applicationoriented services or basemap services, as suggested in section 2.4.

\subsection{Atomic data service \& Design of service}

For developing atomic services, we have to first select the theme of the dataset, then analyze its characteristics in different situations, and then choose the appropriate standard to publish the data. The contexts of the developed service are described by the various types of standardized keywords proposed in section 2. After analysis, a service based on single theme of data may result in a number of services with different contexts due to the different consideration of "situational description" and "visualization". For example, we choose to use the keywords of "convenience store" to describe the theme of the dataset of convenience store. (Table 3) We then find some convenience store is open 24-hrs, while some of them are only open in daytime, so we decide to develop two services respectively for these two situations. Both of them are described by the situation keyword of "rainy" because they sell umbrella whenever the store is open. Finally, we decide to publish the data in both WMS and WFS. The former is designed to meet the direct map overlay purpose, while the latter is designed to provide the location of the features, so that application developers can modify the map symbols (e.g., replaced by the symbol of umbrella).

\begin{tabular}{|l|l|l|}
\hline \multicolumn{1}{|c|}{ Service name } & Service standard & \multicolumn{1}{c|}{ keywords } \\
\hline $\begin{array}{l}\text { Convenience } \\
\text { store }\end{array}$ & WFS, WMS & day, rain \\
\cline { 2 - 3 } & & all day, rain \\
\hline
\end{tabular}

Table 3. Atomic service for the convenience store.

Table 4 summarizes some of the map services and their contexts implemented in this research.

\begin{tabular}{|c|c|c|}
\hline Service name & Service standard & keywords \\
\hline \multirow{2}{*}{$\begin{array}{l}\text { Convenience } \\
\text { store }\end{array}$} & \multirow{8}{*}{ WFS, WMS, } & day, rain \\
\hline & & all day, rain \\
\hline \multirow{3}{*}{ Entertainment } & & day, rain \\
\hline & & night, rain \\
\hline & & all day, rain \\
\hline \multirow{2}{*}{$\begin{array}{l}\text { Household life } \\
\text { store }\end{array}$} & & day, rain \\
\hline & & all day, rain \\
\hline Fire station & & all day \\
\hline
\end{tabular}

\begin{tabular}{|c|c|}
\hline Government & day \\
\hline School & day \\
\hline Library & day \\
\hline Park & all day \\
\hline Gas station & all day \\
\hline \multirow{2}{*}{ Gym } & day \\
\hline & Time period \\
\hline
\end{tabular}

Table 4. Implemented atomic services in this research.

\subsection{Basemap service}

Basemap services are typically used as a reference map for showing the neighbourhood information about the region of interests. For particular applications, more themes of map services can be superimposed onto the basemap, as long as their contexts are compatible. In the following discussion, the implementation of basemap service include day map and night map. The major differences between the two are the selected themes of data and the cartographic design.

(1) Day basemap service (Figure 3)

The day map is published via WMTS, meaning the illustrated phenomena (selected themes of data) may change at different levels. The valid time is setup to be between the period of 6 A.M. to 6 P.M., such that any request during this time period will receive the response from the day map service.

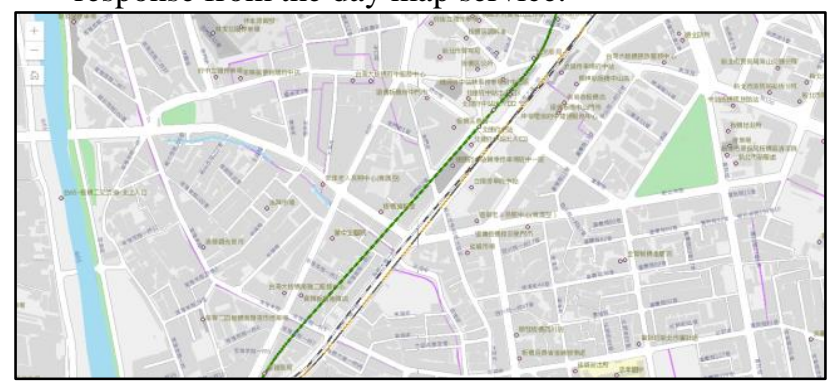

Figure 3. BaseMap (Day).

(2) Night basemap service (Figure 4) Based on the same set of datasets, the night map service adopts a different cartographic style. To emulate the visual effect of night, the colors chosen to present background are mainly grey or black. Road networks represented by the color of white. The symbols of street lights are highlighted by the color of yellow.

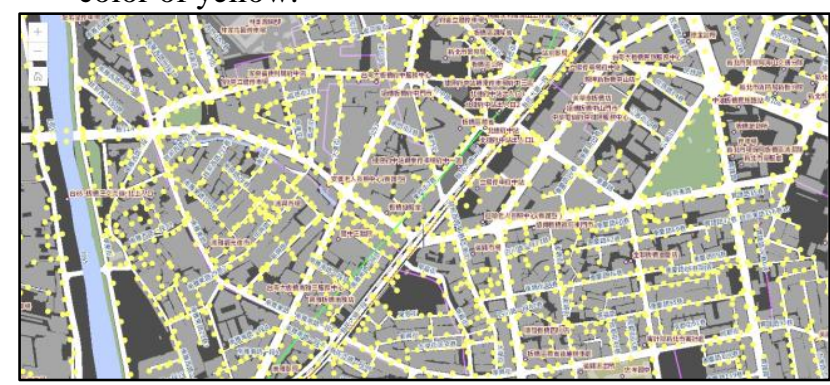

Figure 4. BaseMap (Night). 


\subsection{Application oriented map service}

(1) Rainy night map

Suppose the current situation is at night and rainy, the useful information for users may include the shops selling rain gear, the shelters or places they can temporarily stay, etc. We can develop a "rainy map service" (an application-oriented services) by selecting map services that provide the context of "rainy." Based on the above discussion, all the map services described by the "situation keyword" of "rainy" can be selected. However, some of the map services are only valid during daytime, so an additional constraint on the "situation keyword" of "night" or "all day" are added. For better geospatial reference, the night basemap service is also selected. Application developers can choose either WMS or WFS for presenting the selected map services. When WFS is used, the map symbols from different map services can be modified to meet this particular illustration need.

Table 5 and Figure 5 show the selected map services and the illustration of rainy map service for night, while Table 6 and Figure 6 show the rainy map service for daytime. Note the criteria for selecting map services in these two situations are different.

\begin{tabular}{|l|l|}
\hline \multicolumn{1}{|c|}{ Service name } & \multicolumn{1}{c|}{ keywords } \\
\hline Convenience store & all day, rain \\
\hline \multirow{2}{*}{ Entertainment } & night, rain \\
\cline { 2 - 2 } & all day, rain \\
\hline Household life store & all day, rain \\
\hline
\end{tabular}

Table 5. Selected map services for rainy map (night).

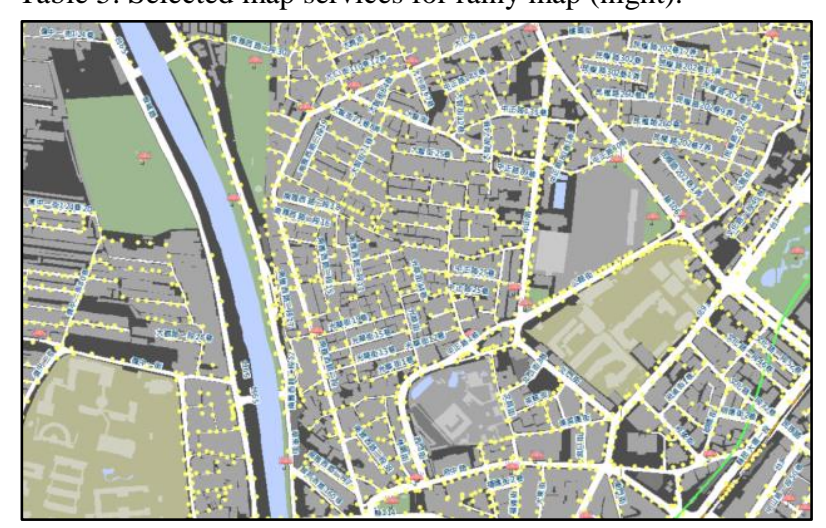

Figure 5. Rainy Map (Night).

\begin{tabular}{|l|l|}
\hline \multicolumn{1}{|c|}{ Service name } & \multicolumn{1}{c|}{ keywords } \\
\hline \multirow{2}{*}{ Convenience store } & day, rain \\
\cline { 2 - 2 } Entertainment & all day, rain \\
\hline \multirow{2}{*}{ Household life store } & day, rain \\
\cline { 2 - 2 } & all day, rain \\
\cline { 2 - 2 } & day, rain \\
\hline
\end{tabular}

Table 6. Selected map services for rainy map (day).

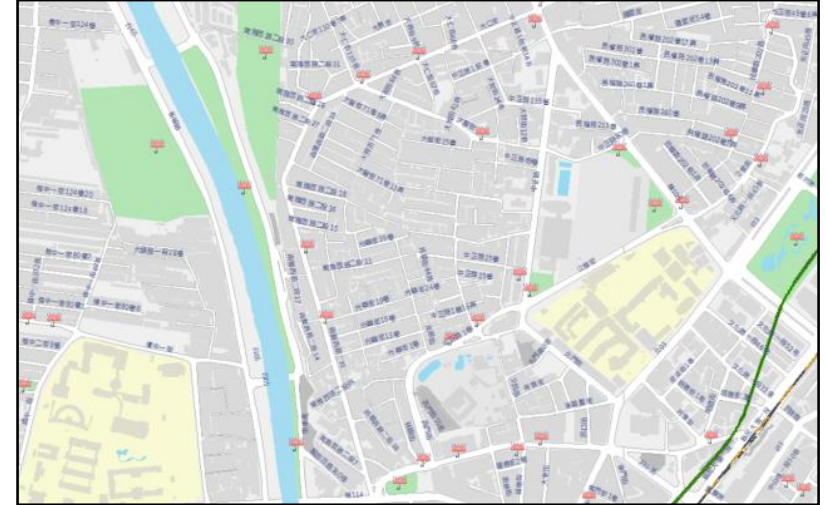

Figure 6. Rainy Map (Day).

\section{(2) Toilet Map}

While looking for toilets in the daytime, the qualified map services may widely include the 24-hour shops, household living stores, gym, entertainment, schools, libraries, parks, government, fire stations, etc. A map service selection rule similar to the rainy map service is defined. The situation keyword of "day" or "all day" are used for finding toilets available at the daytime. Figure 7 shows the results by directly accessing the WMS services, so that the map symbols are predetermined by the original map services. Table 7 shows the selected map services. Figure 8 shows the illustrated results by accessing the WFS service and modifying all the map symbols to the familiar toilet symbol. Figure 9 and Table 8 show the result of the night toilet map. Note only those map services whose context is "all day' or "night" are selected.

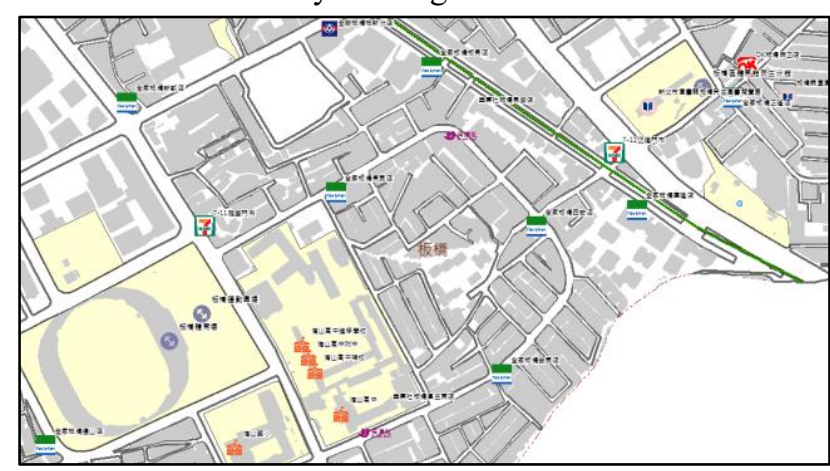

Figure 7. Toilet Map (Originally).

\begin{tabular}{|l|l|}
\hline \multicolumn{1}{|c|}{ Service name } & \multicolumn{1}{c|}{ keywords } \\
\hline \multirow{2}{*}{ Convenience store } & day, rain \\
\cline { 2 - 2 } & all day, rain \\
\hline \multirow{2}{*}{ Entertainment } & day, rain \\
\cline { 2 - 2 } & all day, rain \\
\hline Household life store & day, rain \\
\hline Fire station & all day \\
\hline Government & day \\
\hline School & day \\
\hline Library & day \\
\hline Park & all day \\
\hline Gas station & all day \\
\hline
\end{tabular}




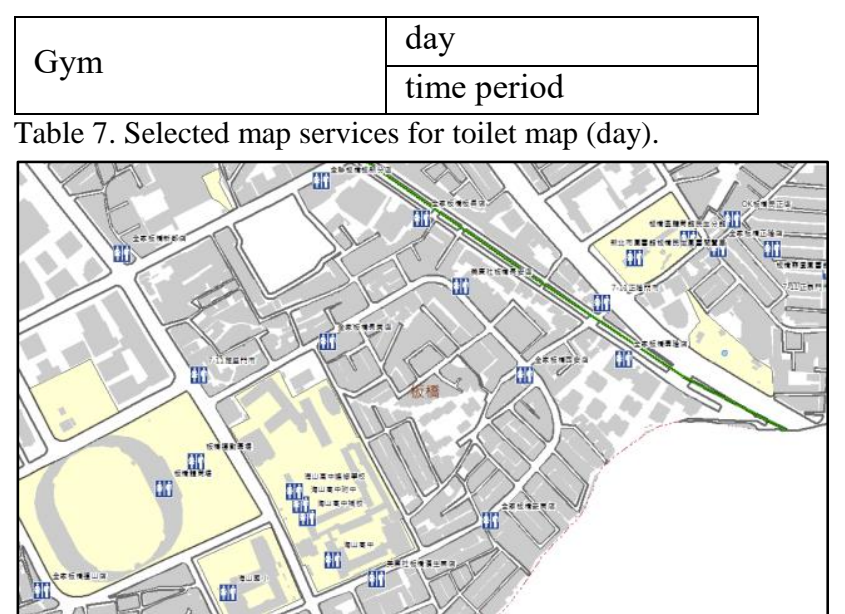

Figure 8. Toilet Map (Day).

\begin{tabular}{|l|l|}
\hline \multicolumn{1}{|c|}{ Service name } & \multicolumn{1}{c|}{ keywords } \\
\hline Convenience store & all day, rain \\
\hline \multirow{2}{*}{ Entertainment } & night, rain \\
\cline { 2 - 3 } & all day, rain \\
\hline Household life store & all day, rain \\
\hline Fire station & all day \\
\hline Park & all day \\
\hline Gas station & all day \\
\hline Gym & time period \\
\hline
\end{tabular}

Table 8. Selected map services for toilet map (night).

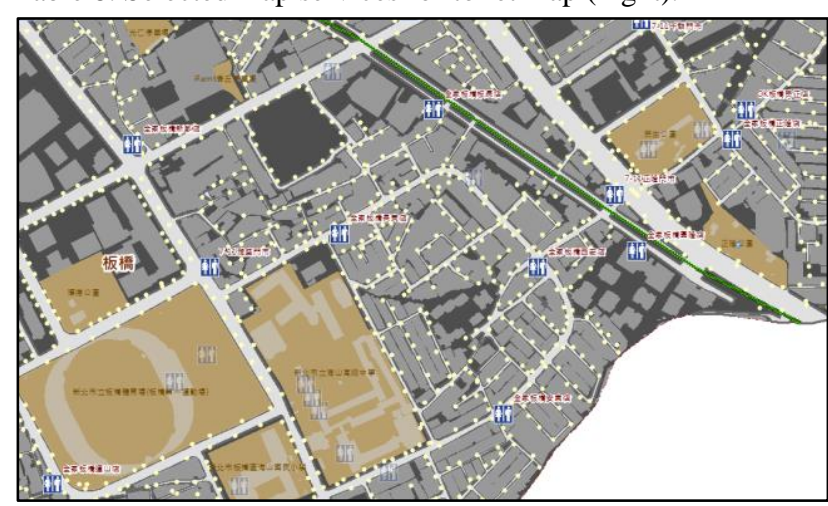

Figure 9. Toilet Map (Night).

\section{Conclusion}

Internet map services have become indispensable utilities and supports to our daily lives. While we enjoy the luxury of visually inspecting the map illustration for making decisions, seldom are we aware of the fact service content has rather limited capabilities to adapt to the phenomena changes in reality. This research proposes a context-driven approach for improving the awareness of situations of the map services. The context analysis procedure and standardized description keywords for individual map services provide a new insight for domain service providers to re-examine the contexts of their services, e.g., map services specifically developed for different consideration of theme/time/environment/transportation. As each services are designed with specific contexts and formally described by the consensus set of keywords, the various map services from different providers serve as a solid framework from which application developers can comprehensively understand, select, combine and mashup various map services to meet particular situation needs. All map services are openly accessible via OGC map service standards for transparent and interoperable usages, some of them are even served with cartographic design according to their contexts. Furthermore, we can switch to access map services with the correct context according to the situations in reality, such that the map service becomes situational-aware. When compared with the current services, the proposed approach provides distinguished advantages of a more flexible and collaborated geospatial web service environment and easier dialogues between application developers and domain service providers, such that map services with specific contexts can be created once and used many times. Finally, the proposed framework is expandable by taking more types of situations into consideration and enriching the contexts of the map services.

\section{Acknowledgement}

Partial results of this paper are from the research project funded by the Ministry of Science and Technology (No. 107-2813-C-006-121-M).

\section{References}

Blefari-Melazzi, N., Casalicchio, E. and Salsano, S. Context-aware Service Discovery in Mobile Heterogeneous Enviroments, Procedings IEEE Mobile and Wireless Communications Summit, 2007, 16th IST, September 2007 pp. 1-5, Budapest, doi: 10.1109/ISTMWC.2007.4299226.

José Rodríguez1 , Maricela Bravo2, Rafael Guzmán1. (2013). Multidimensional Ontology Model to Support Context- ware Systems. Activity Context-Aware System Architectures: Papers from the AAAI 2013 Workshop

Brian P. Walawender, Matt W. Davis. (2010). Development of web-based GIS applications for decisions support and situational awareness.

Michael Batty, Andrew Hudson-Smith, Richard Milton \& Andrew Crooks. (2010). Map mashups, Web 2.0 and the GIS revolution. Annals of GIS.

Buqing Cao ; Jianxun Liu ; Mingdong Tang ; Zibin Zheng ; Guangrong Wang. (2013). Mashup Service Recommendation Based on User Interest and Social Network. 2013 IEEE 20th International Conference on Web Services.

OGC. (2005). OGC Web Map Service - Proposed Animation Service Extension. (OGC 06-045rl).

OGC. (2007). Styled Layer Descriptor profile of the Web Map Service Implementation Specification. (OGC 05078r4)

Harish Chandra Karnatak, Reedhi Shukla, Vinod Kumar Sharma , Y.V.S. Murthy \& V. Bhanumurthy. (2012). Spatial mashup technology and real time data integration in geo-web application using open source GIS - a case study for disaster management. Geocarto International, pages 499-514. 